\title{
Christian Doppler Laboratory: Contextual Interfaces
}

\author{
David Wilfinger, Alexander Meschtscherjakov, Astrid Weiss, and Manfred Tscheligi \\ Christian Doppler Laboratory "Contextual Interfaces", ICT\&S Center, University of \\ Salzburg, Sigmund Haffner Gasse 18, 5020 Salzburg, Austria \\ firstname. lastnamedsbg.ac.at
}

\begin{abstract}
The Christian Doppler Laboratory for Contextual Interfaces is a cooperative research lab dealing with interaction designs of contextual interfaces in the automotive area and the cleanroom of a semiconductor factory. This paper describes the research approach and example activities conducted in the laboratory.
\end{abstract}

\section{Introduction}

Human-Computer Interaction (HCI) is dependent on the concrete characteristics of the context, in which the interaction takes place. To design interfaces for a specific usage context with the aim of improving user experience (UX), it is necessary to identify relevant context factors and understand the particular environment in a holistic way. For that purpose, we established a laboratory with the aim of investigating contextual interfaces on a theoretical level, as well as applying these abstract results into two particular contexts: the factory and the car. In comparison to traditional environments these contexts have been rather neglected in HCI research. The so-called Christian Doppler Laboratory for Contextual Interfaces is located at the Center for Advanced Studies and Research on Information and Communication Technologies (ICT\&S) at the University of Salzburg, Austria. The laboratory is structured into three main areas: a basic research area, a context factory area, and a context car area. This paper gives a short overview of the organizational entities of the laboratory, as well as some example results.

\section{Research Approaches and Results}

In the focus of ongoing activities in the laboratory is the understanding of the two interaction contexts in terms of related UX and contextual influences, as well as a methodological framework to investigate these particular contexts. In the following paragraphs the research areas are introduced with example results.

The basic research area deals with theoretical challenges of context, UX and related methodology. It delivers basic building blocks for contextual UX research and guides their application in the car and the factory context. Within basic research activities we work on UX and context models extending existing approaches for their use in both the car and factory context. These models are informed by basic research studies. As an example we developed car experience characters within the automotive context such as "car as a tool" and "car for self-appreciation" by conducting a content 
analysis of posts in an online car forum. As a second part of basic research, methods to investigate contextual interactions (such as probing and experience sampling) were extended and applied for the specific context of the factory (e.g. cleanroom restrictions) and the car (e.g. safety related issues).

The context factory research is a cooperation between the laboratory and Infineon Technologies Austria AG. It specifically addresses the cleanroom of a semiconductor factory from a user-centered perspective. In the focus of the factory research is the optimization of the workplace for operators (and subsequently an increase of productivity) by introducing new interfaces, such as ambient technology or collaborative human-robot interfaces into this interaction space. All activities are based on a thorough understanding of the context factory and its situated user experiences. To achieve this, we applied qualitative methods in the cleanroom in cooperation with our industrial partner. Ethnographic studies were conducted investigating, equipments, working routines, and social structure of operators. Other studies, including contextual inquiries aimed at understanding UX related to equipment maintenance, occurring context factors in the clean room, the effect of error messages on the workers, and operators' attitudes to robots.

The context car research is a cooperation with AUDIO MOBIL Elektronik GmbH, a tier 1 supplier for the automotive industry. Within the research activities in this context, the car is divided into three design spaces that are of interest for usercentered design: the driver space, the front seat passenger space, and the rear seat passenger space. Together with our industrial partner we aim at understanding the car as an interaction environment combining users in all three spaces. Research in context car includes the influence of particular context factors on drivers' UX. Widening the focus of car HMI research, an ethnographic study aimed at collaboration between drivers and front seat passengers, investigating where assistance can be supported or taken over by technology. Additionally we used cultural probes to understand the usage of a rear seat area by families. Similar to the factory context, interaction studies aimed at understanding UX that evolves out of the usage of a certain system. For that purpose we investigated a multifunctional steering wheel including a touch screen on the steering wheel, cockpit interaction modalities and eco-friendly driver interfaces.

\section{Conclusions and Future Work}

This work gives a very short overview on example activities within a research laboratory planned for seven years. Due to the organization of the laboratory we were able to conduct both basic and applied research in the car and factory context. The development of theoretical approaches within the basic module supported the research conducted in the two contexts. Within the next years we will develop and study alternative interaction designs for both the car and the factory. The financial support by the Federal Ministry of Economy, Family and Youth and the National Foundation for Research, Technology and Development is gratefully acknowledged. 